\title{
Effects of intraoperative PEEP on postoperative pulmonary complications in patients undergoing robot-assisted laparoscopic radical resection for bladder cancer or prostate cancer: study protocol for a randomized controlled trial
}

Zhen-feng Zhou', Jun-biao Fang ${ }^{1}$, Long Chen ${ }^{1}$, Hong-fa Wang ${ }^{1}$, Yong-jian Yu', Wen-yuan Wang ${ }^{1}$, Jia-bao Chen', Miao-zun Zhang ${ }^{2}$ and Shuang-fei $\mathrm{Hu}^{1 *}$

\begin{abstract}
Background: There are increasing studies showing that the use of a lung-protective ventilation strategy has a lung protection effect in patients undergoing abdominal surgery; however, the appropriate positive end-expiratory pressure (PEEP) has not yet defined. Adopting a suitable PEEP may prevent postoperative pulmonary complications. Robot-assisted laparoscopic surgery is the newest and most minimally invasive treatment for bladder cancer or prostate cancer. It is also necessary to consider the effects of Trendelenburg position with pneumoperitoneum on airway pressure and pulmonary function. The role of PEEP during the intraoperative period in preventing postoperative pulmonary complications for robot-assisted laparoscopic surgery is not clearly defined.

Methods/design: A total of 208 patients undergoing robot-assisted laparoscopic radical resection for bladder cancer or prostate cancer will be enrolled and then randomly assigned to a standard PEEP $\left(6-8 \mathrm{~cm} \mathrm{H}_{2} \mathrm{O}\right)$ group and a low PEEP $\left(\leq 2 \mathrm{~cm} \mathrm{H_{2 }} \mathrm{O}\right)$ group. Both groups will receive an inspired oxygen fraction of 0.50 and a tidal volume of $8 \mathrm{~mL} / \mathrm{kg}$ ideal body weight. Standard perioperative fluid management standardization and analgesic treatments will be applied in both groups. The primary endpoint is postoperative pulmonary complications within 7 days after surgery. Secondary endpoints are the modified clinical pulmonary infection score, postoperative extrapulmonary complications, postoperative surgical complications, intensive care unit length of stay, hospital length of stay, and 30-day mortality.

Discussion: This trial aimed to assess the effects of low tidal volumes combined with intraoperative PEEP ventilation strategy on postoperative pulmonary complications in patients undergoing robot-assisted laparoscopic radical resection for bladder cancer or prostate cancer.
\end{abstract}

Trial registration: ID: ChiCTR1800019867. Registered on December 2, 2018.

Keywords: Positive end-expiratory pressure, Postoperative pulmonary complications, Robot-assisted surgery

\footnotetext{
* Correspondence: hushuangfei77@sina.com

${ }^{1}$ Department of Anesthesiology, Zhejiang Provincial People's Hospital

(People's Hospital of Hangzhou Medicine College), Hangzhou 315000, China

Full list of author information is available at the end of the article
}

(C) The Author(s). 2019 Open Access This article is distributed under the terms of the Creative Commons Attribution 4.0 International License (http://creativecommons.org/licenses/by/4.0/), which permits unrestricted use, distribution, and reproduction in any medium, provided you give appropriate credit to the original author(s) and the source, provide a link to the Creative Commons license, and indicate if changes were made. The Creative Commons Public Domain Dedication waiver (http://creativecommons.org/publicdomain/zero/1.0/) applies to the data made available in this article, unless otherwise stated. 


\section{Background}

Robot-assisted laparoscopic surgeries, including robot-assisted laparoscopic radical prostatectomy (RALP) and robot-assisted laparoscopic radical cystectomy (RARC), are the newest and most minimally invasive treatments for bladder cancer or prostate cancer $[1,2]$. The incidence of postoperative pulmonary complications (PPCs) in patients undergoing general surgery is about $5 \%$, and $12 \%$ to $58 \%$ of patients undergoing abdominal surgery will develop a PPC [3, 4]. Furthermore, PPCs are strongly associated with prolonged postoperative hospital stays and a higher risk of mortality [5-7].

Nearly $30 \%$ of surgery patients undergoing general anesthesia and mechanical ventilation are at intermediate to high risk for PPCs, according to large cohort studies $[4,8]$. Both alveolar overstretching and atelectasis induce the release of inflammatory mediators, leading to lung and systemic organ damage [9]. Lung-protective ventilation, including the use of low tidal volumes and positive end-expiratory pressure (PEEP), aims to prevent atelectasis and improve gas exchange $[10,11]$. Furthermore, PEEP has been found to reduce mortality in patients with acute respiratory distress syndrome [12].

Adopting an appropriate PEEP may prevent PPCs. When high PEEP is applied, alveolar may be overinflated and pulmonary vascular resistance is likely to increase; however, use of low PEEP may not prevent atelectasis [9]. A number of studies have shown that, compared with non-protective mechanical ventilation without PEEP, the use of a lung-protective ventilation strategy has a lung-protective effect in patients with healthy lungs who are undergoing abdominal surgery, reducing the incidence of PPC [13, 14]. Although all of these studies recommend the use of a low tidal volume [9, 13-17], the appropriate PEEP has not yet been defined. A multicenter observational study showed that about $20 \%$ of patients did not receive PEEP during routine anesthetic practice [16]. In the Intraoperative Protective Ventilation (IMPROVE) trial, a lung-protective ventilation strategy with lower tidal volumes and a PEEP of 6 $\mathrm{cm} \mathrm{H}_{2} \mathrm{O}$ was associated with improved clinical outcomes in patients undergoing major abdominal surgery [13]. Furthermore, a protective ventilation strategy with a PEEP of $10 \mathrm{~cm} \mathrm{H}_{2} \mathrm{O}$ improved respiratory function and reduced the modified clinical pulmonary infection score (mCPIS) in another study including patients undergoing abdominal non-laparoscopic surgery [14]. However, another study showed that a low tidal volume combined with a low PEEP $\left(3 \mathrm{~cm} \mathrm{H}_{2} \mathrm{O}\right)$ ventilation may increase the risk of PPCs during major surgery such as hepatectomy [17]. In the PROVHILO trial, a ventilation strategy of high PEEP $\left(12 \mathrm{~cm} \mathrm{H}_{2} \mathrm{O}\right)$ did not reduce the incidence of PPCs but more likely caused hemodynamic instability in patients undergoing open abdominal surgery [15].
Therefore, the authors suggested a ventilation strategy of a low tidal volume combined with a low PEEP $(\leq 2 \mathrm{~cm}$ $\left.\mathrm{H}_{2} \mathrm{O}\right)$.

It should be noted that these studies included only open surgeries or various types of abdominal surgery; they did not include patients planning to undergo robot-assisted laparoscopic surgery. Furthermore, it is necessary to consider the effects of steep Trendelenburg (sT) positioning and pneumoperitoneum (PnP) on airway pressure and pulmonary function [18], which can increase intra-abdominal pressure and enhance the cranial displacement of the diaphragm. This displacement will decrease lung compliance and lung volumes and increase lung resistance. Adopting a PEEP of $7 \mathrm{~cm} \mathrm{H}_{2} \mathrm{O}$ during RALP, which could improve arterial oxygenation without causing excessive peak airway pressure, has been recommended [19]. Also, a lung-protective ventilation strategy with a lower tidal volume $\left(\mathrm{V}_{\mathrm{t}}\right)$ of $6 \mathrm{~mL} / \mathrm{kg}$ and a PEEP of $8 \mathrm{~cm} \mathrm{H}_{2} \mathrm{O}$ was associated with less impaired postoperative pulmonary functions in patients undergoing RALP in another recent study [20].

The role of PEEP during the intraoperative period in preventing PPCs for robot-assisted laparoscopic surgery has not been clearly defined. This study may further improve our knowledge regarding the effects of intraoperative PEEP on PPCs, survival rates, and in-hospital stays in patients undergoing robot-assisted laparoscopic radical resection for bladder cancer or prostate cancer.

\section{Methods/design}

\section{Objectives of the study}

This trial aimed to compare the effects of low tidal volumes combined with standard PEEP $\left(6-8 \mathrm{~cm} \mathrm{H}_{2} \mathrm{O}\right)$ with those of low PEEP $\left(\leq 2 \mathrm{~cm} \mathrm{H}_{2} \mathrm{O}\right)$ in patients at risk for complications undergoing robot-assisted laparoscopic radical resection for bladder cancer or prostate cancer during general anesthesia in terms of (1) PPCs; (2) mCPIS, postoperative extrapulmonary complications, changes in chest x-ray findings, and oxygenation; (3) intraoperative complications, including hypoxemia, hypotension, and massive transfusion; and (4) postoperative surgical complications, intensive care unit (ICU) lengths of stay, hospital lengths of stay, and 30-day mortality.

\section{Study design}

This unfunded, parallel-group, double-blinded, prospective, randomized controlled clinical trial was registered at http://www.chictr.org.cn (ChiCTR1800019867) and was conducted at the Department of Anesthesiology and Intensive Care of Zhejiang Provincial People's Hospital. The first patient will be randomly assigned in January 2019. This trial protocol is conducted in accordance with the Consolidated Standards of Reporting Trials 
(CONSORT) guidelines (Fig. 1). The SPIRIT (Standard Protocol Items: Recommendation for Interventional Trials) 2013 Checklist is presented in Additional file 1.

\section{Blinding, data collection, randomization, and record- keeping \\ Selection of the participants}

Researchers will be trained prior to investigation. Study data, including patient clinical characteristics, intraoperative respiratory parameters, postoperative outcomes, and laboratory tests, will be recorded in case report forms (CRFs) (Additional file 2).

An independent researcher will randomly assign the participants to the study group (standard PEEP group) and control group (low PEEP group) in a ratio of 1:1. The random sequence will be computer-generated, and participants will be allocated in numerical order with sealed opaque envelopes. The attending anesthesiologist performs anesthesia strictly in accordance with the research protocol and is also responsible for data during the preoperative, intraoperative, and post-anesthesia care unit (PACU) period. The chief surgeon performs the postoperative laboratory testing. An independent researcher will be involved in postoperative follow-up and data collection. Statistical analysis will be performed by a statistician who does not participate in the data collection. Patients, research staff, surgeons, ICU physicians, and the statistician will be unaware of the group allocation. Some preoperative characteristics and laboratory results will be automatically derived from a computer database.

The original data (CRF and relevant records) will be maintained for 10 years and then destroyed in accordance with hospital standards.

\section{Selection of the participants}

Patients scheduled for elective robot-assisted laparoscopic radical resection for bladder cancer or prostate

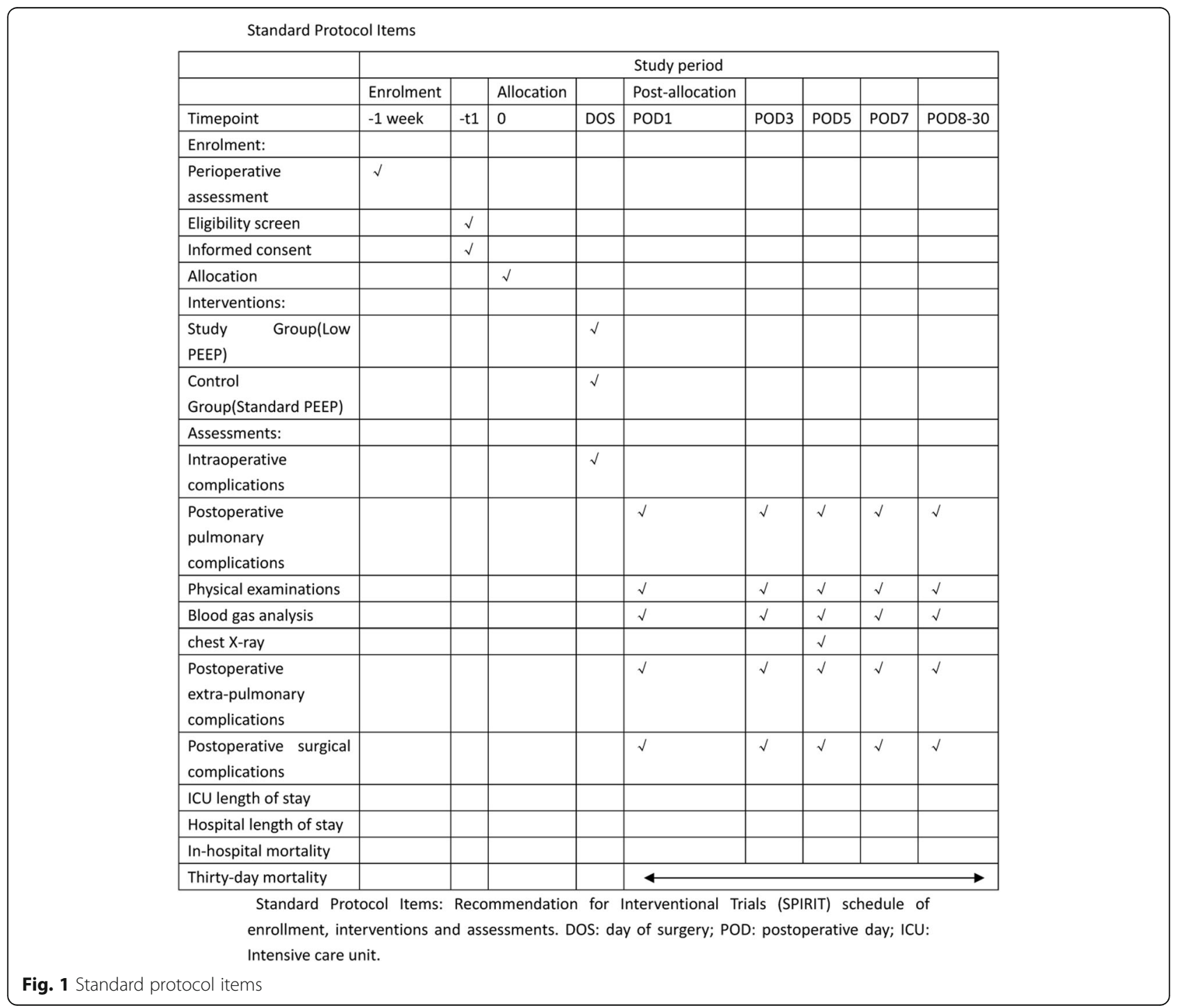


cancer under general anesthesia will be screened and recruited during preoperative assessment. Patients meeting inclusion criteria will be required to provide written informed consent. The participant can withdraw from the trial at any time.

Inclusion criteria are patients older than 18 years, American Society of Anesthesiologists (ASA) physical status I-III, and body mass index (BMI) between 18 and 35 $\mathrm{kg} / \mathrm{m}^{2}$. Exclusion criteria are listed as following: emergency surgery or history of lung surgery; history of mechanical ventilation within the 2 weeks before recruitment; non-invasive ventilation or oxygen therapy at home; acute respiratory failure (pneumonia, acute lung injury, or acute respiratory distress syndrome); history of chronic obstructive pulmonary disease, persistent hemodynamic instability, or severe cardiac disease (New York Heart Association class III or IV, persistent ventricular tachyarrhythmias, or acute coronary syndrome); sepsis or septic shock; need for continuous renal replacement therapy; progressive neuromuscular illness; pregnancy; participation in another study; or refusal to participate.

\section{Time course of the study}

\section{Preoperative admission}

Medical history, ASA physical status, BMI, 12-lead electrocardiogram (ECG), laboratory results, chest x-ray or computed tomography (CT) scan, ARISCAT (Assess Respiratory Risk in Surgical Patients in Catalonia) score (the ARISCAT study; Additional file 3), nutritional risk screening (NRS 2002 tool), and the results of echocardiography and spirometry (in cases of history of coronary artery disease or smoking) will be recorded.

\section{Intraoperative care}

A central venous catheter and an arterial cannula will be placed before induction of anesthesia. Peripheral oxygen saturation $\left(\mathrm{SpO}_{2}\right)$, arterial blood pressure, heart rate, ECG, end-tidal carbon dioxide tension $\left(\mathrm{EtCO}_{2}\right)$, and bispectral index (BIS) will be monitored continuously. PnP, tidal volume, PEEP, airway pressures including peak pressure and plateau pressure, airway resistance (Raw), dead space fraction (Vds/Vt), core temperature, and arterial blood gas analysis data will be recorded.

Crystalloid (12-15 mL/kg per hour) is infused to maintain hemodynamic stability and a central venous pressure of $5-12 \mathrm{~cm} \mathrm{H}_{2} \mathrm{O}$. Blood loss and vasodilation are supplemented by colloidal fluid.

Routine anesthesia is induced with intravenous dexmedetomidine $(1 \mu \mathrm{g} / \mathrm{kg})$ or midazolam $(0.05-0.075 \mathrm{mg} / \mathrm{kg})$, cisatracurium $(2 \mathrm{mg} / \mathrm{kg})$, propofol $(2-3 \mathrm{mg} / \mathrm{kg})$, and fentanyl $(1-3 \mu / \mathrm{kg})$ for tracheal intubation. Anesthesia is maintained with propofol, sevoflurane, and remifentanil infusion to maintain the BIS of $40-50$ until skin suturing is completed. Cisatracurium $(1.0-1.5 \mathrm{mg} / \mathrm{kg})$ is administered every hour and is adjusted in accordance with the anesthesiologist's decision.

Ropivacaine is administrated as local anesthetic before and at the end of operation. Fentanyl $(1-3 \mu \mathrm{g} / \mathrm{kg})$ and flurbiprofenaxetil $50 \mathrm{mg}$ are required before remifentanil is stopped.

\section{Postoperative care}

Patients will be transferred to the PACU after surgery regardless of whether they are still intubated. Postoperative pain management will be suggested to achieve a visual analogue scale (VAS) pain score of less than 3 out of 10 using a patient-controlled intravenous analgesia pump including fentanyl $(0.3-0.5 \mu \mathrm{g} / \mathrm{kg})$, flurbiprofenaxetil $(100 \mathrm{mg})$, and palonosetron hydrochloride $(0.25 \mathrm{mg})$ palazidine.

The ICU physician and surgeon will independently monitor clinical progress and all endpoints by daily physical examinations. Appropriate prophylactic antibiotics and antithrombotic treatments will be administered as required during the postoperative period. Chest $\mathrm{x}$-ray or CT scanning will be performed when clinically indicated. Arterial blood gas analysis will be performed on postoperative day 1 (POD 1) and POD 3 , and other laboratory tests will be performed on POD 1, POD 3, POD 5, and POD 7. The examinations will be repeated and microbiology tests will be performed when the development of pulmonary complications is suspected.

\section{Study arms and intraoperative ventilation protocol}

Patients will be randomly assigned to the low PEEP ventilation group (PEEP $\leq 2 \mathrm{~cm} \mathrm{H}_{2} \mathrm{O}$ ) or the standard PEEP group ( $\mathrm{PEEP}=6-8 \mathrm{~cm} \mathrm{H}_{2} \mathrm{O}$ ) using a volume-controlled ventilation strategy (Datex Ohmeda S/5 Avance; GE Healthcare, Helsinki, Finland) with a tidal volume of 8 $\mathrm{mL} / \mathrm{kg}$ ideal body weight (IBW), an inspired oxygen fraction $\left(\mathrm{FiO}_{2}\right)$ of 0.50 , and an inspiratory-to-expiratory ratio of 1:2. Respiratory rate should be adjusted to maintain $\mathrm{EtCO}_{2}$ between 35 and $45 \mathrm{~mm} \mathrm{Hg}$, and plateau pressure should be no more than $30 \mathrm{~cm} \mathrm{H}_{2} \mathrm{O}$. IBW is calculated as follows [13]: $45.5+0.91 \times$ (centimeters of height 152.4) for females and $50+0.91 \times$ (centimeters of height - 152.4) for males. Recruitment maneuvers (RMs) [21] will be performed immediately after tracheal intubation and every time the ventilator is interrupted until the end of surgery in each group. The compliance of the respiratory system will be calculated as $V_{t}$ / (plateau pressure of the respiratory system - PEEP).

RMs will be performed as follows:

1. Pressure support ventilation (PSV) mode

2. PEEP set to $30 \mathrm{~cm} \mathrm{H}_{2} \mathrm{O}$

3. Inspiratory gas flow set to the highest value

4. Duration of the maneuver $=30 \mathrm{~s}$. 
A rescue therapy will be applied in case of desaturation (defined as a peripheral $\mathrm{SpO}_{2}$ of less than $92 \%$ ), consisting of increased $\mathrm{FiO}_{2}$ to $100 \%$ in each group and increasing PEEP in the low PEEP group (Additional file 4).

\section{Study endpoints \\ Primary outcome measure}

The primary endpoint is PPCs, including new atelectasis or infiltrates on a chest $\mathrm{x}$-ray or CT scanning, respiratory failure defined as the need for non-invasive or invasive ventilation or partial pressure of arterial oxygen/fraction of inspired oxygen $\left(\mathrm{PaO}_{2} / \mathrm{FiO}_{2}\right)$ of less than 300 within 7 days after surgery [21].

\section{Secondary outcome measures}

Secondary outcome variables are any pulmonary complications and extrapulmonary complications as follows (Additional file 5):

1. Intraoperative complications: pneumothorax confirmed by chest $\mathrm{x}$-ray and any other complications.

2. PPCs within 30 days after surgery. Those PPCs are scored in accordance with a grading scale ranging from 0 to 4 [22] (grade 0 representing no PPCs and grades 1 to 4 representing gradually worse forms of PPCs) within 7 and 30 days after surgery (Table 1).

3. PPCs will also be analyzed separately (Table 1 ).

a. Pneumonia is defined in accordance with criteria of the Centers for Disease Control and Prevention [23];

b. Purulent sputum;

c. Postoperative hypoxemia and severe hypoxemia [24]; d. Suspected pulmonary infection is described in a previous study [15];

e. Pulmonary infiltrate is defined in accordance with consensus guidelines [25]: chest $\mathrm{x}$-ray demonstrating monolateral or bilateral infiltrate.

f. Atelectasis, pleural effusion, and pneumothorax are identified by chest $\mathrm{x}$-ray.

g. The mCPIS is calculated as previously described [26] (Table 2).

h. Suspected pulmonary complications [14];

i. Requirement for postoperative ventilation (respiratory failure that requires non-invasive or invasive ventilation or both) at any time after surgery in accordance with standard criteria and clinical practice guidelines [22].

4. Postoperative extrapulmonary complications within 30 days after surgery:

a. Criteria for systemic inflammatory response syndrome [12]

b. Sepsis and severe sepsis [12]

c. Septic shock [12]

d. Other extrapulmonary infection including surgical site infection (SSI) [27] and intraabdominal abscess

e. Need for postoperative blood transfusion

f. Postoperative surgical complications: anastomotic leakage and need for surgical reintervention, defined in accordance with consensus criteria [28]

g. Unexpected ICU admission or readmission

h. ICU length of stay and hospital length of stay

i. Hospital-free days at follow-up day 30

j. In-hospital mortality and 30-day mortality (all-cause mortality 30 days after randomization).

Table 1 Grade scale for postoperative pulmonary complications

\begin{tabular}{|c|c|}
\hline $\begin{array}{l}\text { Grade } \\
\text { scale }\end{array}$ & Detailed description \\
\hline Grade 1 & $\begin{array}{l}\text { - Cough, dry } \\
\text { - Microatelectasis: abnormal lung findings and temperature greater than } 37.5^{\circ} \mathrm{C} \text { without other documented cause; results of chest } \\
\text { radiograph indicates no new findings as compared to pre-operative result } \\
\text { - Dyspnea, not due to other documented cause }\end{array}$ \\
\hline Grade 2 & $\begin{array}{l}\text { - Cough, productive, not due to other documented cause } \\
\text { - Bronchospasm: new wheezing or pre-existent wheezing resulting in change of therapy } \\
\text { - Hypoxemia } \\
\text { - Atelectasis: radiological confirmation plus either a temperature of more than } 37.5^{\circ} \mathrm{C} \text { or abnormal lung findings } \\
\text { - Hypercarbia, transient, requiring treatment, such as naloxone or increased manual or mechanical ventilation }\end{array}$ \\
\hline Grade 3 & $\begin{array}{l}\text { - Pleural effusion, resulting in thoracentesis } \\
\text { - Pneumonia, suspected: radiological evidence without bacteriological confirmation } \\
\text { - Pneumonia, proven: radiological evidence and documentation of pathological organism by Gram stain or culture } \\
\text { - Pneumothorax } \\
\text { - Re-intubation postoperative or intubation, period of ventilator dependence (non-invasive or invasive ventilation) of not more than } 48 \mathrm{~h}\end{array}$ \\
\hline Grade 4 & $\begin{array}{l}\text { Ventilatory failure: postoperative non-invasive ventilation dependence of at least } 48 \mathrm{~h} \text { or re-intubation with subsequent period of } \\
\text { ventilator dependence of at least } 48 \mathrm{~h}\end{array}$ \\
\hline
\end{tabular}


Table 2 The definition of modified clinical pulmonary infection score

\begin{tabular}{llll}
\hline Items & CPIS points & & \\
\cline { 2 - 4 } & 0 & 1 & Abundant + purulent \\
\hline Tracheal secretions & Rare & Abundant & Localized \\
Chest $x$-ray infiltrates & No infiltrate & Diffused & $\leq 36.5$ or $\geq 39.0$ \\
Temperature, ${ }^{\circ} \mathrm{C}$ & $36.5-38.4$ & $38.5-38.9$ & $<4000$ or $>11,000+$ band forms $\geq 500$ \\
Leukocyte count, per $\mathrm{mm}^{3}$ & $4000-11,000$ & $<4000$ or $>11,000$ & $\leq 240$ and no evidence of ARDS \\
$\mathrm{PaO}_{2} / \mathrm{FiO}_{2}, \mathrm{~mm} \mathrm{Hg}$ & $>240$ or ARDS & & Positive \\
\hline Microbiology & Negative & & \\
\hline
\end{tabular}

Abbreviations: ARDS Acute respiratory distress syndrome, CPIS clinical pulmonary infection score, $\mathrm{PaO}_{2} / \mathrm{FiO}_{2}$ Partial pressure of arterial oxygen/fraction of inspired oxygen

From postoperative day 7 (POD 8 to POD 30, follow-up) Secondary endpoints and any mortality will also be evaluated during the follow-up period. The CONSORT flowchart of the trial is shown in Fig. 2.

\section{Data monitoring and handling of implausible values or missing values}

A clinical investigator will identify implausible values. Missing continuous variables should be less than $10 \%$ and will be replaced by median. Missing values will be replaced by the mean of all plausible data (both groups) of the respective endpoint. Data monitoring is managed by an independent investigator who is not involved in the study. The progress of the study will be evaluated, and the completeness and accuracy of the data (informed consent forms, source data, CRFs, and outcome variables) will be verified.

\section{Statistics}

Normally distributed variables will be expressed as the mean \pm standard deviation and will be compared with the Student's $t$ test. Categorical variables will be compared by using the chi-squared test or Fisher's exact test. Non-normal continuous variables will be expressed as median (interquartile range) and evaluated with the MannWhitney $U$ test. Intention-to-treat analyses are performed to compare the composite outcome measure at 7 days in the two groups by the chi-squared test (or Fisher's exact test as appropriate), and multiple logistic regression analysis adjusting will be performed to identify various risk factors (for the primary outcome and the pulmonary complications at POD 30). Adjusted analyses are performed with the use of robust Poisson generalized-linear-model regression for continuous outcomes and are presented as relative risks with $95 \%$ confidence intervals. A $P$ value of less than 0.05 will be considered statistically significant, and all reported $P$ values will be two-sided. Interim analysis of safety will be conducted after enrollment of the first 200 patients. All analyses will be conducted by using SPSS version 18.0 (SPSS, Chicago, IL, USA) software.

\section{Sample size calculation}

The incidence rate of PPCs is 39\% in the low PEEP group [15]. The two-tailed chi-squared test is performed, and we estimate that 188 patients are required to provide $90 \%$ power to detect a $50 \%$ relative difference between the two groups (39\% to $20 \%$ ), and type I error probability was 0.05 . Given that the follow-up lost rate is 10\%, 208 cases in total are needed. Analysis is computed by using G-Power (version 3.1; Informer Technologies, Inc. Universität Kiel, Germany).

\section{Adverse events and interruption of the trial}

All patients will be continuously monitored during the study, including daily visits during in-hospital and daily phone-call visits during the out-of-hospital follow-up period (until POD 30). All serious adverse, unexpected, or possibly related events will be recorded in the CRF and will be reported to the data monitoring and safety committee (DMSC). DMSC can recommend that the study should be stopped unless there is evidence that patient is safety (a between-group difference in serious adverse events or in 30-day mortality is found).

\section{Discussion}

In this pragmatic, prospective, randomized controlled trial of patients undergoing robot-assisted laparoscopic radical resection for bladder cancer or prostate cancer, our aim will be to assess not only possible single effects of PEEP levels on major PPCs from those of lower tidal volumes and RM but also relevant clinical parameters associated with alterations in pulmonary function, such as chest $\mathrm{x}$-ray, abnormalities, mCPIS, arterial oxygenation/peripheral oxygen saturation in air, and changes in dyspnea/cough/secretions. Our findings might change the current practice of mechanical ventilation in patients undergoing robot-assisted laparoscopic radical resection for bladder cancer or prostate cancer.

Notably, mechanical ventilation itself is one of the major contributors to PPCs [29]. sT positioning together with pneumoperitoneum is also an important risk factor for PPCs [30]. Intra-abdominal pressure is frequently higher than airway pressure during PnP 


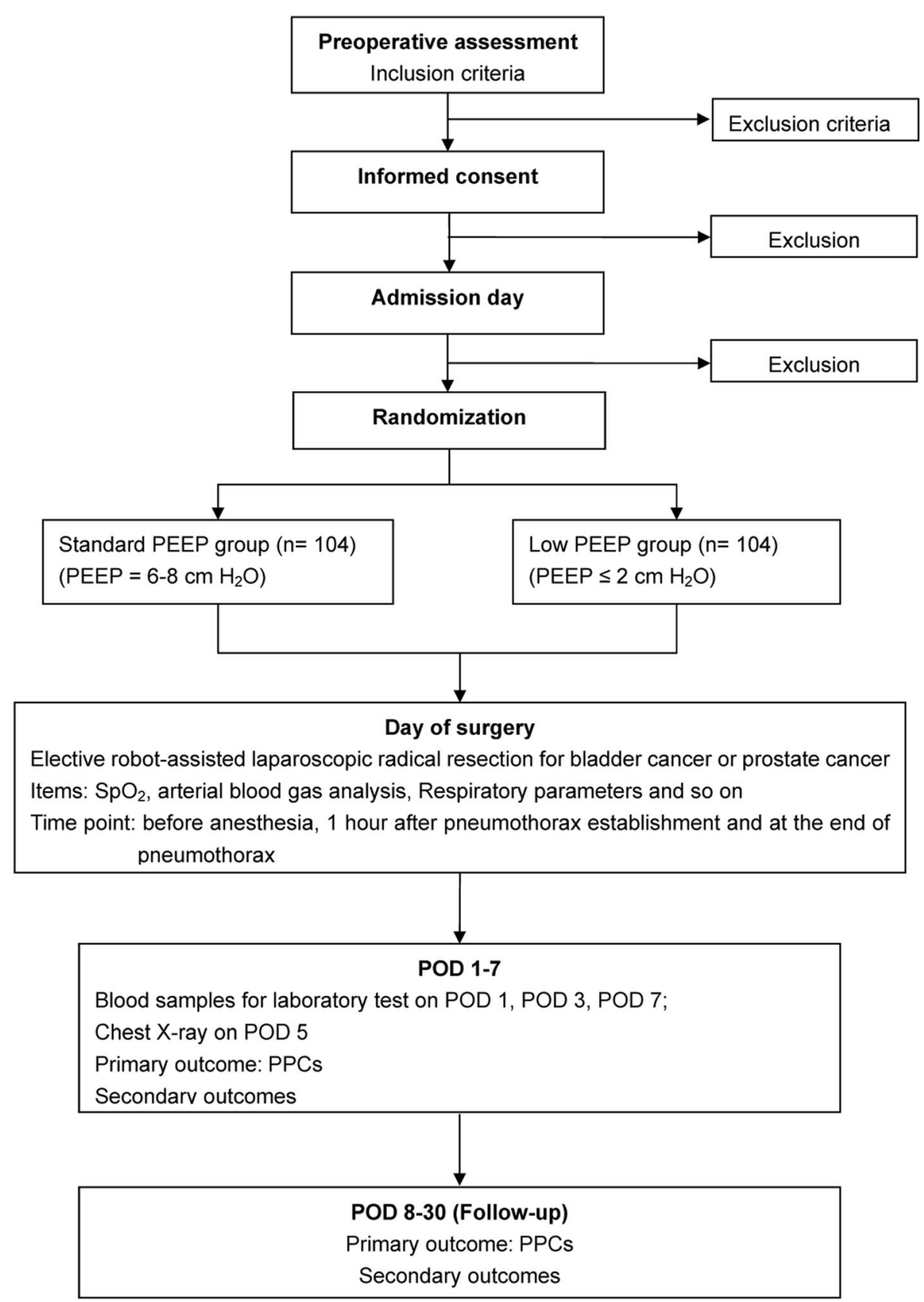

Fig. 2 The CONSORT (Consolidated Standards of Reporting Trials) flowchart of the trial

with carbon dioxide $\left(\mathrm{CO}_{2}\right)$ for laparoscopic surgery. This pressure gradient usually causes cephalad displacement of the diaphragm and collapses adjacent pulmonary tissues. PnP also decreases respiratory compliance and arterial oxygenation [31]. All of these influences on PnP lead finally to atelectasis [32]. The major difference between robot-assisted surgeries and other laparoscopic surgeries is the sT positioning, which will further decrease respiratory compliance and vital capacity.
On the other hand, PEEP is thought to prevent the development of atelectasis by keeping the airways open and maintaining adequate gas exchange at the end of the expiratory period during PnP [9]. Certainly, the level of PEEP should be adopted according to the patient's and surgical characteristics as well as to the patient's positioning.

The optimal PEEP has not yet been defined in patients undergoing robot-assisted laparoscopic surgery, even though adopting a PEEP of over $5 \mathrm{~cm} \mathrm{H}_{2} \mathrm{O}$ in patients 
undergoing laparoscopic surgery is recommended [11]. One study recommended adopting a PEEP of $7 \mathrm{~cm} \mathrm{H}_{2} \mathrm{O}$ during RALP [19], and another recent study found that a PEEP of $8 \mathrm{~cm} \mathrm{H}_{2} \mathrm{O}$ was the optimal level of PEEP in patients undergoing RALP [20]. As we know that low levels of PEEP are potentially associated with atelectasis by promoting repeated opening and closing of small airways [33]. However, higher levels of PEEP may increase mean airway pressure of the respiratory system and likely even impair hemodynamics.

There is an increasing number of highly qualitative randomized controlled trials regarding intraoperative mechanical ventilation and PPCs in both abdominal surgeries [10, 11] and laparoscopic surgeries [32], whereas direct assessment of the effect in patients undergoing robot-assisted laparoscopic radical resection for bladder cancer or prostate cancer is still lacking. The potential significance of this trial is that it may provide evidence of the effects of intraoperative PEEP on PPCs in patients undergoing robot-assisted laparoscopic radical resection for bladder cancer or prostate cancer.

There are some potential strengths of the present trial protocol. First, the included patients will undergo elective robot-assisted laparoscopic radical resection for bladder cancer or prostate cancer with longer anesthesia duration, which is a potential risk factor for PPCs [7]. Second, this trial design includes instructions for fluid management standardization and analgesic treatments during the perioperative period. Third, the adopting ARISCAT score is considered the most valuable tool for predicting PPCs, although various scores have been developed for predicting PPC incidence based on various countries and surgical populations [9].

\section{Trial status}

The study protocol version number is V1.0 (September 10, 2018). It is first submitted to the ethics committee of Zhejiang Provincial People's Hospital (People's Hospital of Hangzhou Medicine College) on September 10, 2018, and finally approved on October 22, 2018 (Additional files 6 and 7). The first participant is expected to be recruited before March 2019, and the estimated completion date of recruitment is October 2021.

\section{Additional files}

Additional file 1: SPIRIT (Standard Protocol Items: Recommendation for Interventional Trials) 2013 Checklist: Recommended items to address in a clinical trial protocol and related documents. (DOC $123 \mathrm{~kb}$ )

Additional file 2: Case report form. (DOC $274 \mathrm{~kb}$ )

Additional file 3: Preoperative risk index of postoperative pulmonary complications by ARISCAT (Assess Respiratory Risk in Surgical Patients in Catalonia) score. (DOC $40 \mathrm{~kb}$ )
Additional file 4: Strategy for oxygen saturation $\left(\mathrm{SpO}_{2}\right)$ decreasing. (DOC 28 kb)

Additional file 5: Definitions of study endpoints. (DOC $42 \mathrm{~kb}$ ) Additional file 6: Appendix Figure study institutional review board (IRB) in original language. (TIF $390 \mathrm{~kb}$ )

Additional file 7: Appendix Figure study institutional review board (IRB) in English. (TIF $401 \mathrm{~kb}$ )

\section{Abbreviations}

ARISCAT: Assess Respiratory Risk in Surgical Patients in Catalonia; ASA: American Society of Anesthesiologists; BIS: Bispectral index; BMI: Body mass index; CONSORT: Consolidated Standards of Reporting Trials; CRF: Case report form; CT: Computer tomography; DMSC: Data monitoring and safety committee; ECG: Electrocardiogram; $\mathrm{EtCO}_{2}$ : End-tidal carbon dioxide tension; $\mathrm{FiO}_{2}$ : Fraction of inspired oxygen; IBW: Ideal body weight; ICU: Intensive care unit; mCPIS: Modified clinical pulmonary infection score; PACU: Postanesthesia care unit; PEEP: Positive end-expiratory pressure;

PnP: Pneumoperitoneum; POD: Postoperative day; PPC: Postoperative pulmonary complication; RALP: Robot-assisted laparoscopic radical prostatectomy; RM: Recruitment maneuver; $\mathrm{SpO}_{2}$ : Oxygen saturation; sT: Steep Trendelenburg; $V_{T}$ : Tidal volume

\section{Acknowledgments}

We thank all of the contributors and collaborators for their support in this study and all of the participating patients.

\section{Funding}

This trial is conducted with no external or internal funding. The tests in this trial are performed as routine medical care in our institute.

\section{Availability of data and materials \\ Not applicable.}

\section{Authors' contributions}

ZFZ and SFH designed the study protocol and wrote the manuscript. HFW and MZZ designed the statistical method. The work of patient recruitment and data collecting will be carried out by JBF, YJY, WYW, LC, and JBC. SFH is the study director and JBF is the principal investigator of this study. All authors have read and approved the manuscript.

\section{Authors' information}

Not applicable.

\section{Ethics approval and consent to participate}

The study was approved by the ethics committee of Zhejiang Provincial People's Hospital (People's Hospital of Hangzhou Medicine College) (registration number KY2018027) on October 22, 2018. Any subsequent protocol and informed consent document amendments must be approved by the designated member of the ethics committee. All communications with the regulatory authorities and the ethics committee must be recorded. All recruited patients will be informed of the trial purposes and their duties within the trial before randomization. Informed consent will be obtained from all study participants. Recruited patients can withdraw from the study at any time without providing any specific reason. The patient data will be stored in a separate, safe place but may be reviewed by the relevant investigator.

Consent for publication

Not applicable.

\section{Competing interests}

The authors declare that they have no competing interests.

\section{Publisher's Note}

Springer Nature remains neutral with regard to jurisdictional claims in published maps and institutional affiliations. 


\section{Author details}

'Department of Anesthesiology, Zhejiang Provincial People's Hospital (People's Hospital of Hangzhou Medicine College), Hangzhou 315000, China. ${ }^{2}$ Department of General Surgery, Ningbo Medical Center Lihuili Hospital, Ningbo 325000, China.

Received: 16 December 2018 Accepted: 15 April 2019

Published online: 29 May 2019

\section{References}

1. Trinh QD, Sammon J, Sun M, Ravi P, Ghani KR, Bianchi M, et al. Perioperative outcomes of robot-assisted radical prostatectomy compared with open radical prostatectomy: results from the nationwide inpatient sample. Eur Urol. 2012;61:679-85

2. Menon $M_{1}$ Hemal AK. Vattikuti Institute prostatectomy: a technique of robotic radical prostatectomy: experience in more than 1000 cases. J Endourol. 2004;18:611-9 discussion 619.

3. Canet J, Gallart L, Gomar C, Paluzie G, Vallès J, Castillo J, et al. Prediction of postoperative pulmonary complications in a population-based surgical cohort. Anesthesiology. 2010;113:1338-50.

4. Arozullah AM, Khuri SF, Henderson WG, Daley J. Development and validation of a multifactorial risk index for predicting postoperative pneumonia after major noncardiac surgery. Ann Intern Med. 2001;135:847-57.

5. Shander A, Fleisher LA, Barie PS, Bigatello LM, Sladen RN, Watson CB. Clinical and economic burden of postoperative pulmonary complications: patient safety summit on definition, risk-reducing interventions, and preventive strategies. Crit Care Med. 2011;39:2163-72.

6. Khuri SF, Henderson WG, DePalma RG, Mosca C, Healey NA, Kumbhani DJ. Determinants of long-term survival after major surgery and the adverse effect of postoperative complications. Ann Surg. 2005;242:326-41 discussion 341-3.

7. Smetana GW, Lawrence VA, Cornell JE. Preoperative pulmonary risk stratification for noncardiothoracic surgery: systematic review for the American College of Physicians. Ann Intern Med. 2006;144:581-95.

8. Arozullah AM, Daley J, Henderson WG, Khuri SF. Multifactorial risk index for predicting postoperative respiratory failure in men after major noncardiac surgery. The National Veterans Administration Surgical Quality Improvement Program. Ann Surg. 2000;232:242-53.

9. Güldner A, Kiss T, Serpa Neto A, Hemmes SN, Canet J, Spieth PM, et al Intraoperative protective mechanical ventilation for prevention of postoperative pulmonary complications: a comprehensive review of the role of tidal volume, positive end-expiratory pressure, and lung recruitment maneuvers. Anesthesiology. 2015;123:692-713.

10. Sutherasan $Y$, Vargas M, Pelosi P. Protective mechanical ventilation in the non-injured lung: review and meta-analysis. Crit Care. 2014;18:211.

11. Futier E, Constantin JM, Pelosi P, Chanques G, Kwiatkoskwi F, Jaber S, et al. Intraoperative recruitment maneuver reverses detrimental pneumoperitoneum-induced respiratory effects in healthy weight and obese patients undergoing laparoscopy. Anesthesiology. 2010;113:1310-9.

12. Brower RG, Matthay MA, Morris A, Schoenfeld D, Thompson BT, Wheeler A Ventilation with lower tidal volumes as compared with traditional tida volumes for acute lung injury and the acute respiratory distress syndrome. N Engl J Med. 2000;342:1301-8.

13. Futier E, Constantin JM, Paugam-Burtz C, Pascal J, Eurin M, Neuschwander A, et al. A trial of intraoperative low-tidal-volume ventilation in abdominal surgery. N Engl J Med. 2013;369:428-37.

14. Severgnini P, Selmo G, Lanza C, Chiesa A, Frigerio A, Bacuzzi A, et al. Protective mechanical ventilation during general anesthesia for open abdominal surgery improves postoperative pulmonary function. Anesthesiology. 2013;118:1307-21.

15. Hemmes SN, de Abreu MG, Pelosi P, Schultz MJ. High versus low positive end-expiratory pressure during general anaesthesia for open abdominal surgery (PROVHILO trial): a multicentre randomised controlled trial. Lancet. 2014;384:495-503.

16. Jaber $\mathrm{S}$, Coisel $Y$, Chanques $\mathrm{G}$, Futier E, Constantin JM, Michelet $P$, et al. A multicentre observational study of intra-operative ventilatory management during general anaesthesia: tidal volumes and relation to body weight. Anaesthesia. 2012;67:999-1008.

17. Sato H, Nakamura K, Baba Y, Terada S, Goto T, Kurahashi K. Low tidal volume ventilation with low PEEP during surgery may induce lung inflammation. BMC Anesthesiol. 2016;16:47.
18. Lestar M, Gunnarsson L, Lagerstrand L, Wiklund P, Odeberg-Wernerman S. Hemodynamic perturbations during robot-assisted laparoscopic radical prostatectomy in 45 Trendelenburg position. Anesth Analg. 2011;113:1069-75.

19. Lee HJ, Kim KS, Jeong JS, Shim JC, Cho ES. Optimal positive end-expiratory pressure during robot-assisted laparoscopic radical prostatectomy. Korean J Anesthesiol. 2013:65:244-50

20. Haliloglu M, Bilgili B, Ozdemir M, Umuroglu T, Bakan N. Low Tidal VolumePositive End-expiratory Pressure versus High Tidal Volume-Zero Positive Endexpiratory Pressure and Postoperative Pulmonary Functions in Robot-assisted Laparoscopic Radical Prostatectomy. Med Princ Pract. 2017;26:573-8.

21. Ruszkai Z, Kiss E, László I, Gyura F, Surány E, Bartha PT, et al. Effects of intraoperative PEEP optimization on postoperative pulmonary complications and the inflammatory response: study protocol for a randomized controlled trial. Trials. 2017;18:375

22. Hulzebos EH, Helders PJ, Favié NJ, De Bie RA, de la Riviere AB, Van Meeteren $\mathrm{NL}$. Preoperative intensive inspiratory muscle training to prevent postoperative pulmonary complications in high-risk patients undergoing CABG surgery: a randomized clinical trial. JAMA. 2006;296:1851-7.

23. Schumacher M, Wangler M, Wolkewitz M, Beyersmann J. Attributable mortality due to nosocomial infections. A simple and useful application of multistate models. Methods Inf Med. 2007;46:595-600.

24. Keenan SP, Sinuff T, Burns KE, Muscedere J, Kutsogiannis J, Mehta S, et al. Clinical practice guidelines for the use of noninvasive positive-pressure ventilation and noninvasive continuous positive airway pressure in the acute care setting. CMAJ. 2011;183:E195-214.

25. Bernard GR, Artigas A, Brigham KL, Carlet J, Falke K, Hudson L, et al. Report of the American-European consensus conference on ARDS: definitions, mechanisms, relevant outcomes and clinical trial coordination. The Consensus Committee. Intensive Care Med. 1994;20:225-32.

26. Fartoukh M, Maitre B, Honoré S, Cerf C, Zahar JR, Brun-Buisson C. Diagnosing pneumonia during mechanical ventilation: the clinical pulmonary infection score revisited. Am J Respir Crit Care Med. 2003;168:173-9.

27. Horan TC, Gaynes RP, Martone WJ, Jarvis WR, Emori TG. CDC definitions of nosocomial surgical site infections, 1992: a modification of CDC definitions of surgical wound infections. Infect Control Hosp Epidemiol. 1992;13:606-8.

28. Dindo D, Demartines N, Clavien PA. Classification of surgical complications: a new proposal with evaluation in a cohort of 6336 patients and results of a survey. Ann Surg. 2004;240:205-13.

29. Mascia L, Pasero D, Slutsky AS, Arguis MJ, Berardino M, Grasso S, et al. Effect of a lung protective strategy for organ donors on eligibility and availability of lungs for transplantation: a randomized controlled trial. JAMA. 2010;304: 2620-7.

30. Reinius H, Jonsson L, Gustafsson S, Sundbom M, Duvernoy O, Pelosi P, et al. Prevention of atelectasis in morbidly obese patients during general anesthesia and paralysis: a computerized tomography study. Anesthesiology. 2009;111:979-87.

31. Hazebroek EJ, Haitsma JJ, Lachmann B, Bonjer HJ. Mechanical ventilation with positive end-expiratory pressure preserves arterial oxygenation during prolonged pneumoperitoneum. Surg Endosc. 2002;16:685-9.

32. Park SJ, Kim BG, Oh AH, Han SH, Han HS, Ryu JH. Effects of intraoperative protective lung ventilation on postoperative pulmonary complications in patients with laparoscopic surgery: prospective, randomized and controlled trial. Surg Endosc. 2016;30:4598-606.

33. Duggan M, Kavanagh BP. Pulmonary atelectasis: a pathogenic perioperative entity. Anesthesiology. 2005;102:838-54.

\section{Ready to submit your research? Choose BMC and benefit from:}

- fast, convenient online submission

- thorough peer review by experienced researchers in your field

- rapid publication on acceptance

- support for research data, including large and complex data types

- gold Open Access which fosters wider collaboration and increased citations

- maximum visibility for your research: over $100 \mathrm{M}$ website views per year

At $\mathrm{BMC}$, research is always in progress.

Learn more biomedcentral.com/submission 